CSID Working Paper Series

\#CSID_WP_2012-008

\title{
An Agent-based Model Based on Field Experiments
}

\author{
Marco A. Janssen \\ Arizona State University, USA
}

July 25, 2012

The Center for the Study of Institutional Diversity resides in the School of Human Evolution and Social Change at Arizona State University. CSID can be found on the internet at: http://csid.asu.edu/. CSID can be reached via email at csid@asu.edu or via phone at (480)965-8318.

(C)2012 by M.A. Janssen. All rights reserved. 


\section{An Agent-based Model Based on Field Experiments}

Marco A. Janssen ${ }^{\mathrm{a}}$,

${ }^{\mathrm{a}}$ Center for the Study of Institutional Diversity, Arizona State University, Tempe, AZ 85287;

Corresponding author:

Marco A. Janssen

School of Human Evolution and Social Change, Arizona State University

PO Box 872402

Tempe, AZ 85287-2402, USA

Marco.Janssen@asu.edu; Phone: 480965 4193; Fax: 4809657671 


\section{Introduction}

This chapter describes the empirical calibration of a theoretical model based on data from field experiments. Field experiments on irrigation dilemmas were performed to understand how resource users overcome asymmetric collective action problems (Janssen et al., 2012). The fundamental problem facing irrigation systems is how to solve two related collective action problems: 1) the provision of the physical and ecological infrastructure necessary to utilize the resource (water), and 2) the irrigation dilemma where the relative positions of "head-enders" and "tail-enders" generate a sequential access to the resource itself (water). If the actors behave as rational, self-interested agents, it is difficult to understand how irrigation infrastructure could ever be constructed and maintained by the farmers who utilize the system as opposed to a government irrigation bureaucracy. Wittfogel (1957) argued that a centralized control was indispensable for the functioning of larger irrigation systems and hypothesized that some state-level societies have emerged as a necessary side-effect of solving problems associated with the use of large-scale irrigation

Even if the initial problem of providing the infrastructure were solved, water that is available to the head-enders may not necessarily be shared with the tail-enders, as long as the head-enders have a positive marginal return on the use of water. The vulnerability of irrigation system performance to the behavior of selfinterested rational actors leads to the question of why so many self-organized irrigation systems exist and persist for so long (Hunt, 1988; Lansing, 1991; Ostrom, 1992).

The field experiments held in Colombia and Thailand show that trust and fairness are key components that drive the decision making. The more groups experience inequality in contributions and collection of the common resource in previous rounds, the more likely that they will lower their contributions. The fact that some groups were irrigators while others were students had no significant effect on the decisions. We use the experimental data to develop a decision theoretic model of the irrigation dilemma. The model is calibrated on the data from 32 experiments. The resulting model can be used to define new hypotheses and design new field experiments.

\section{Experimental design}

The field experiments were designed to be implemented in the field with participants who manage natural resources in their daily lives (see also Janssen et al., 2012). In the irrigation game participants have positions A, $\mathrm{B}, \mathrm{C}, \mathrm{D}$ or E. A has the first choice to harvest water from the common resource. Then B has the next turn to harvest water from whatever amount was left by $\mathrm{A}$, and so on. The location of the five players is randomly determined before the first round and remains fixed over the first set of ten rounds of the game. Participants receive an endowment $\omega$ of 10 tokens in each round. First each participant makes a decision $x_{i}$ on how much to invest in a public fund that generates the infrastructure and therefore determines the amount of water available for the whole group to share. In Table 1, the water provision generated is defined as a function $\mathrm{f}()$ of the total investments of the five participants.

Table 1. Water production as a function of units invested in the public infrastructure.

\begin{tabular}{ll}
\hline Total units invested by all 5 players & Water available \\
\hline $0-10$ & 0 \\
$11-15$ & 5 \\
$16-20$ & 20 \\
$21-25$ & 40 \\
$26-30$ & 60 \\
$31-35$ & 75 \\
$36-40$ & 85 \\
$41-45$ & 95 \\
$46-50$ & 100 \\
\hline
\end{tabular}


Second, each player, in sequential turns from upstream to downstream players decides how much to extract from the water available to her, that is, the water produced minus the water extracted by those before her in the sequence. Each token kept (not invested) in the first stage has a monetary value for the player that is equal to the value of each unit of water extracted in the second stage.

This experiment includes a first dilemma of upstream participants who need the contribution of downstream participants to maintain the structure of their common resource, which is crucial for the production of water in the game. However, the downstream participants can only obtain benefits from the resource if upstream participants avoid the temptation to deplete the common resource and leave little water for downstream players.

Under this asymmetric game, participants first experience a provision dilemma in the contributions stage, and then face a resource appropriation dilemma when they extract from the generated resource. The earnings of the participants are the result of provision $-x_{i}-$ and extraction $-y_{i}-$ decisions, and the resulting payoff $\mathrm{z}_{\mathrm{i}}$ for player $\mathrm{i}$ is defined as

$z_{i}=\omega-x_{i}+y_{i}$

where

$$
\sum_{i=j+1}^{5} y_{i} \leq f\left(\sum_{i=1}^{5} x_{i}\right)-\sum_{i=1}^{j} y_{i} \text { for } \mathrm{j}=0,1,2,3 \text {, and } 4
$$

If participants were rational self-interested individuals nobody would invest in providing the infrastructure in the first round. Since the upstream participant is expected to collect the whole resource, downstream participants will not invest. For participant A there is no benefit to invest when others don't. If this is the reasoning of the participants in the last round of experiment we find via backward induction that the same happens for all earlier rounds. Thus, the Nash equilibrium for this game is that no one invests and all receive 10 tokens for group earnings of 50 tokens.

To define the cooperative solution we calculate the maximum amount of the infrastructure plus tokens not invested. There are multiple social optimum outcomes. For a 41 tokens investment, a resource of 95 tokens is generated, and for a 46 tokens investment a resource of 100 tokens is generated in each round. The total earnings of the group in the cooperative solution amounts to 104 tokens, doubling the social earnings of the Nash equilibrium.

\section{Model description}

\section{Purpose}

The purpose of the model is to understand which components of a decision theoretical model are most important to explain data from field experiments.

\section{State variables and scales}

The model has 5 agents who make decisions in 10 rounds of an irrigation experiment.

\section{Process overview and scheduling}

Each round all agents first make a decision of how much to invest in the public fund based on expectations and preferences they have. When the level of the resource of the irrigation system is known, agents make decisions on how much to take starting from the upstream agent $\mathrm{A}$ to the downstream agent $\mathrm{E}$. The outcome of the decisions affects the expectations that are used in the following round.

\section{Design concepts}


- Prediction. Agents have expectation on the level of cooperation of others. They have an initial level of expectation which is updated each round.

- Interaction. Agents interact indirectly via decisions on how much to invest in a public fund and how much to extract from a common resource.

- Stochasticity. Decisions are made based on the expected utility of the different options. The option that leads to the highest utility is not automatically chosen since agents make a probabilistic choice using the expected utility of the different options.

\section{Initialization}

The decision theoretical model that is used to define the decisions includes 6 parameters. Each agent has parameter values that are drawn from Gaussian distributions, which are listed in Table 2.

\section{Input}

The model uses two sets of input data. Table 1 defines the production function of the water production. Depending on the investment decisions agents make, a certain level of the common resource - water - is produced.

The second set of data as listed in Table 2 is the set of parameter values of the distributions that define the decision making of the agents. The values of the parameters are determined in the calibration process as described below.

Table 2. Range of parameters and mean and standard deviation of distributions of parameter ranges.

\begin{tabular}{llccc}
\hline Parameter & Description & Range & $\begin{array}{c}\text { Range } \\
\text { Mean }\end{array}$ & $\begin{array}{c}\text { Range } \\
\text { standard deviation }\end{array}$ \\
\hline$\alpha$ & Strength aversion to exploiting others & {$[\beta, 1]$} & {$[-1,1]$} & {$[0,1]$} \\
$\beta$ & Degree of altruistic tendency & {$[-\infty, \alpha]$} & {$[-1,1]$} & {$[0,1]$} \\
$\lambda$ & Parameter to define probabilities & {$[0, \infty]$} & {$[0,5]$} & {$[0,1]$} \\
$\eta$ & Initial level of cooperation of others & {$[0,1]$} & {$[0,1]$} & {$[0,1]$} \\
$\tau_{1}$ & Learning rate investments & {$[0,1]$} & {$[0,1]$} & {$[0,1]$} \\
$\tau_{2}$ & Learning rate extractions & {$[0,1]$} & {$[0,1]$} & {$[0,1]$} \\
\hline
\end{tabular}

\section{Submodels}

\section{Utility}

We assume that agents maximize their utility. This utility $u_{i}$ is formalized in a general way to include different types of other-regarding preferences:

$$
u_{i}=z_{i}-\alpha_{i} \cdot \max \left(z_{i}-\bar{z}_{-i}, 0\right)+\beta_{i} \cdot \max \left(\bar{z}_{-i}-z_{i}, 0\right)
$$

where $\alpha$ and $\beta$ are initially assumed to be the same for all agents, $z_{i}$ is agent i's earnings, and $\bar{z}_{-i}$ is the average earnings of the other agents in the group. $\alpha$ can be regarded as the strength of an individual's aversion to exploiting others, and $\beta$ can be regarded as an individual's degree of altruistic tendency. A lower value of $\beta$ compared to $\alpha$ implies that a player gives a larger weight to his own payoff when his payoff is smaller than the average payoff of others compared to when it is larger. In line with Charness and Rabin (2002), we can define the following cases for $\beta \leq \alpha \leq 1$ :

Case 1: The players like to have their payoffs higher than those of the other players. When $\beta \leq \alpha \leq 0$, the player is highly competitive. 
Case 2: Players prefer the payoffs among all players to be equal. This "Inequity Aversion" holds when $\beta<0<\alpha \leq 1$ (see Fehr and Schmidt 1999).

Case 3: The third model is the so-called "Social Welfare Consideration," which holds when $0<\beta \leq \alpha \leq 1$. The parameter $\alpha$ captures the extent to which a player weighs the average payoffs of the other $n-1$ agents compared to his own payoff, when his own payoff is higher than the average payoff of the others.

Case 4: If $\alpha=\beta=0$, we have the condition in which a player cares only about his or her own welfare.

Investment decision

An agent makes two decisions. First all agents independently decide how much to invest $\mathrm{x}_{\mathrm{i}}$. In order to make this decision, agents are assumed to estimate the expected utility based on the expected behavior of others.

The expected investment level of others is equal to

$$
\hat{x}_{-i}=\omega \cdot 4 \cdot \eta_{i}
$$

where $\eta_{i}$ is the cooperation level of other agents as expected by agent $i$. This enables each agents to estimate an expected level of the public infrastructure, $\hat{p}_{i}$. For each level of investment $\mathrm{x}_{\mathrm{i}}$, the expected level is

$$
\hat{p}_{i}=x_{i}+\hat{x}_{-i}
$$

Agents make a prediction how much of the resource would be available to the group using the production function of Table 1 with the expected value $\hat{p}_{i}$.

How much is expected to be available to agent $\mathrm{i}$ depends on how much upstream agents have taken from $\hat{p}_{i}$. The lower the level of cooperation they expect from the other participants, representing here the upstream participants, the less she expects to receive from the resource before it is her turn. Hence agents assume that an amount $\hat{y}_{i}^{A}$ is available for agent $\mathrm{i}$.

$\hat{y}_{i}^{A}=\hat{p}_{i} \cdot\left(1-\left(\frac{i-1}{5}\right)^{\left(2-\eta_{i}\right)}\right) \cdot$

If agent $i$ expects that other agents are cooperative, $\eta_{i}=1$, they will take an equal share from the resource. If they are expected to be less cooperative, more than an equal share is expected to be taken.

In rounds 2 to 10 a simpler estimation technique is used by the agent to determine $\hat{y}_{i}^{A}$. The agents are assumed to expect the upstream participants take a share $s_{i}$ from the expected resource size.

$\hat{y}_{i}^{A}=\hat{y}_{i}^{A} \cdot s_{i}$

The value of $s_{i}$ is updated each round as defined below:

We use the values of $\alpha_{i}$ and $\beta_{i}$ to define how much the agent takes from the share that is expected to be available to her. Agents who are selfish are expected to take the whole amount of available resources, but those with other-regarding preferences are expected to take a lower level.

$\hat{y}_{i}=\hat{y}_{i}^{A} \cdot\left(1-\alpha_{i}\right) \cdot\left(1-\beta_{i}\right)$ 
Now the agent can define her utility of investing $x_{i}$ and receiving $\hat{y}_{i}$ from the resource. Using the expected earnings, we can estimate the expected utility for agent $i$ for each level of investment. Based on the expected utility levels, agents make a probabilistic choice of how much to invest

$$
\operatorname{Pr}(x)=\frac{\exp (\lambda \cdot u(x))}{\sum_{X} \exp (\lambda \cdot u(X))}
$$

Where $\operatorname{Pr}(\mathrm{x})$ is the probability of investing an amount $\mathrm{x}$ in the public fund and $\lambda$ is the weight given to the utility values. If $\lambda$ is 0 all options have an equal probability, while if $\lambda$ is equal to infinity the agents choose the option with the highest expected utility.

\section{Extraction decision}

Based on the investment decisions of the agents the actual level of the public infrastructure $p$ can be determined. Now, each agent makes a decision how much to collect, based on the available resource at the turn she can make the decision. Similarly to the investment decisions, the expected utility for each level of collection is determined, and decisions are made from upstream to downstream.

\section{Learning}

The agents update the expected level of cooperation $\eta_{i}$ based on the information they received on the average investments of the other agents. The learning parameter $\tau_{1}$ defined the speed of learning. If $\tau_{1}$ is equal to 1 , agents do not learn, while if $\tau_{1}$ is equal to 0 agents assume that the level of cooperation in the next round is the same as observed in the current round.

$$
\eta_{i}=\eta_{i} \cdot \tau_{1}+\left(1-\tau_{1}\right) \cdot \frac{\bar{x}_{-i}}{10}
$$

Similar for the expected share that upstream agents are expected to extract, we assume that agents update the value of $s_{i}$ based on the observed share, where $\tau_{2}$ is a learning rate.

$$
s_{i}=s_{i} \tau_{2}+\left(1-\tau_{2}\right) \frac{y_{i, t-1}}{p_{t-1}}
$$

\section{Overview model parameterization}

Within the model parameterization framework this is case 9 . We do not have a large $\mathrm{N}$ to simulate, since we only have 5 participants in each experiment. The field work does not cover $100 \%$ of the population since we use data from a sample of the populations from a few communities. We have done field work in the community and focus our model on a few behaviors.

M1: To define the model we make use of theory, especially rational choice theory with modifications based on behavioral game theory (Camerer, 2003). We assume agents learn, have other-regarding preferences, and have a trembling hand when making decisions. These assumptions are confirmed by the interviews and the statistical analysis of the experimental data (Janssen et al. 2012).

M2: Attributes of the agents are based on theory and observations, namely ability to learn and having otherregarding preferences. We do not distinguish social-demographic variables since they were not found to have significant impact in the statistical analysis of the experimental analysis. As such the attributes of the agents are derived from the calibration process itself. 
M3: The method to collect behavioral data is the performance of field experiments.

M4. Each agent has a unique parameter setting, which is the result of parameter distributions. The parameter distributions are the outcome of the calibration process. For simplicity's sake we assumed truncated normal distributions. The data did not suggest exploring bimodal or other multi-model distributions. However, we acknowledge that alternative assumptions on agent types could be used to calibrate the model.

M5: The model is not scaled up, although the resulting general model could be used to inform models of irrigation at a watershed level.

\section{Technical details}

\section{Experimental data}

Before we discuss the parameterization of the model, we first discuss the outcomes of the experiments. A detailed analysis of the experimental results can be found in Janssen et al. (2012). Figure 1 shows the average level of contributions to the public fund by all villager and student groups in each round. Figure 1 shows that the average investment is around $50 \%$ and reduces only slightly.

In the Figure 2 we report the average contributions to the infrastructure in each of the locations in the watershed. There is no difference in the level of contributions to the infrastructure among the different locations. However, there is a significant inequality of the extraction levels across participants upstream, A and $\mathrm{B}$, and the participants downstream, $\mathrm{D}$ and $\mathrm{E}$ (Figure 2).

The inequality can also be quantified by gini coefficients. We calculated the gini coefficients for investments as well as extractions. The average gini coefficient for investments is 0.27 and this level does not change significantly. The average gini coefficient for extractions is 0.44 and also here this level does not change significantly.

Janssen et al. (2012) report detailed statistical analysis which shows that the results are not affected by the type of participants, whether they are irrigators, fishers, or forest appropriators, or students. What is affecting the initial level of investments is the trust in other people of the community. The investment levels in subsequent rounds are affected by the level of inequality of the extractions of the common resource.

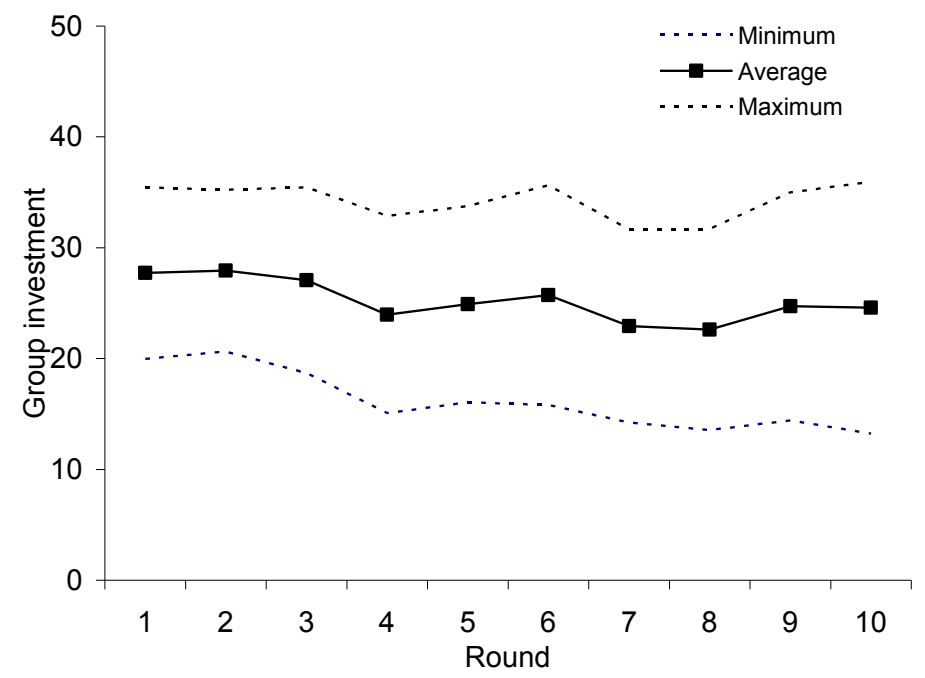

Fig. 1. The average group investments for 10 rounds. The dotted line is the average $+/-$ the standard deviation of the 32 experiments. 


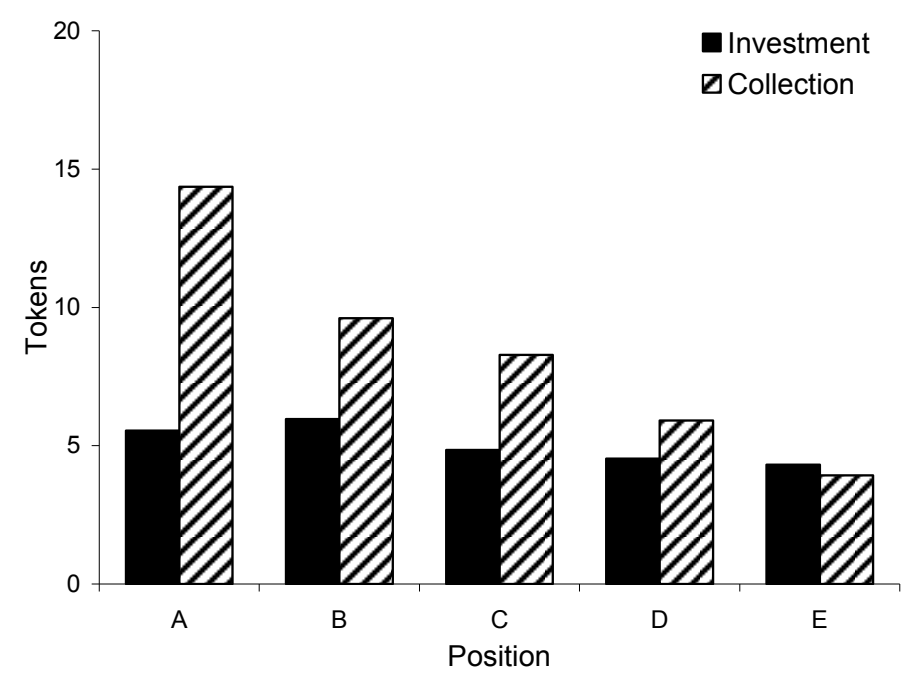

Fig. 2. The average contribution and collection level per round for each position.

\section{Calibration}

We calibrated the model on the experimental data. We used the standard genetic algorithm of BehaviorSearch.org for the model that is implemented in Netlogo 4.1.3. The model code and documentation can be found at http://www.openabm.org/model/3073/version/1. For the fitness evaluation of each parameter configuration we run the model 100 times 32 runs. For each of the 32 runs we compare the simulated statistics with the actual statistics.

The fit between the model and the data is defined as follows. For each of the metrics included, we calculate the fitness score between 0 and 1 , using

$f_{i}=1-\left(d_{e}-d_{s}\right)^{2}$

Where the data of the experiments, $d_{e}$, and simulations, $d_{s}$, are scaled to values between 0 and 1 . Then the fitness values of all 5 metrics are aggregated to derive the final fitness score used in the calibration. We compare the results of three ways of aggregating the information as spelled out below.

The metrics included to evaluate the performance of the model include:

- Average group level investments in the public infrastructure level over the 10 rounds. To calculate $f_{1}$ the simulated average group level investment is denoted by $x_{s i}$ and the average group level investment of the data is denoted by $\bar{x}_{d i}$. Since the maximum group investment level is 50 , the difference is divided by 50 to scale $f_{1}$ between 0 and 1 .

$f_{1}=1-\frac{1}{10} \cdot \sum_{i=1}^{10}\left(\frac{x_{s i}-\bar{x}_{d i}}{50}\right)^{2}$

- The average contribution per position. In the calculation of $f_{2}$ the simulated average level of investments per position over 10 rounds is represented as $x_{s j}^{p p}$ and the data as $\bar{x}_{d j}^{p p}$. To scale $f_{2}$ between 0 and 1 , the difference is divided by 10 since contributions per person are up to 10 tokens.

$f_{2}=1-\frac{1}{5} \cdot \sum_{j=1}^{5}\left(\frac{x_{s j}^{p p}-\bar{x}_{d j}^{p p}}{10}\right)^{2}$

- The average collection per position. In the calculation of $f_{3}$ the simulated average level of collections per position over 10 rounds is represented as $y_{s j}^{p p}$ and the data as $\bar{y}_{d j}^{p p}$. To scale $f_{3}$ between 0 and 1 , the 
difference is divided by 100 since the maximum collection a person can make is 100 tokens (the maximum resource).

$$
f_{3}=1-\frac{1}{100} \cdot \sum_{j=1}^{5}\left(\frac{y_{s j}^{p p}-\bar{y}_{d j}^{p p}}{100}\right)^{2}
$$

- The average gini coeffient of contributions. $G_{s}^{c o n}$ is the gini coefficient of investment levels in a round average of 10 rounds in each game, averaged over all the simulated games. Similarly, $G_{d}^{c o n}$ represented the average gini coefficient per round for all the data.

$f_{4}=1-\left(G_{s}^{c o n}-G_{d}^{c o n}\right)^{2}$

- The average gini coeffient of collected tokens. $G_{s}^{c o l}$ is the gini coefficient of harvested tokens in a round average of 10 rounds in each game, averaged over all the simulated games. Similarly, $G_{d}^{c o l}$ represented the average gini coefficient per round for all the data.

$f_{5}=1-\left(G_{s}^{c o l}-G_{d}^{c o l}\right)^{2}$

There are different ways to aggregate the individual fits with the indicators. We distinguish three ways, and compare the impacts on the calibration of using the different aggregation methods. The first approach is to multiply the fitness scores of the five indicators.

$f^{m l t}=f_{1} \cdot f_{2} \cdot f_{3} \cdot f_{4} \cdot f_{5}$

The second approach is to calculate the average of the fitness scores:

$f^{\text {avg }}=\left(f_{1}+f_{2}+f_{3}+f_{4}+f_{5}\right) / 5$

And finally, the calibration can be evaluated on the minimum level of all five fitness scores.

$f^{\min }=\min \left(f_{1}, f_{2}, f_{3}, f_{4}, f_{5}\right)$

\section{Calibration process}

We first discuss the results of the calibration of the three different fitness functions. Thereafter we perform a sensitivity analysis of the model and present a simpler model that captures the basic results. Our aim is to understand the importance of the basic components of the model and develop a simple model that represents the key empirical features of the model.

When we perform a calibration we run a genetic algorithm with 50 individual randomized starting conditions. We present the parameter values of the 100 best solutions found and the best fitness score. The genetic algorithm, using the standard genetic algorithm from BehaviorSearch 0.72 (beta), has a population size of 50, a mutation rate of 0.01 , and a cross over rate of 0.7 . We stop the genetic algorithm after 1000 fitness evaluations. It is important to note here that the fitness is only calculated for new parameter combinations. Each parameter is distributed using a truncated normal distribution (Table 2). The truncation means that drawn parameter values that are not within the eligible range of the parameter are not used, and a new parameter value is generated.

Table 3 shows the fitness scores for the different aggregation metrics and the different solutions. When agents are selfish and rational and their behavior leads to the Nash equilibrium, the fitness scores are lower compared to the cooperative solution. Hence the behavior of the participants is closer to the cooperative solution compared to the Nash equilibrium. The fitness scores of the results of the calibration are higher. Although the fitness scores are highest for the aggregation function maximized, they are closely together.

Table 4 shows the optimal parameter distributions for the different calibration functions. We use the data from the best solution of the 50 runs of the genetic algorithm. 
The agents have a strong aversion to exploit others $(\alpha)$ and a strong tendency to altruism $(\beta)$. Agents are expecting an initial level of cooperation of about $50 \%$ from the fellow participants. Learning is slow ( $\tau$ value are often higher than 0.50$)$.

Table 3. Fitness scores for the different solutions and for the different aggregation functions.

\begin{tabular}{|c|c|c|c|c|c|}
\hline & $\begin{array}{c}\text { Nash } \\
\text { equilibrium }\end{array}$ & $\begin{array}{c}\text { Cooperative } \\
\text { solution }\end{array}$ & $\begin{array}{c}\text { Max } \\
\mathrm{f}^{\text {mlt }}\end{array}$ & $\begin{array}{c}\text { Max } \\
\mathrm{f}^{\text {avg }}\end{array}$ & $\begin{array}{c}\text { Max } \\
\mathrm{f}^{\text {min }}\end{array}$ \\
\hline $\mathrm{f}^{\text {mlt }}$ & 0.008 & 0.008 & 0.813 & 0.809 & 0.800 \\
\hline $\mathrm{f}^{\text {avg }}$ & 0.329 & 0.427 & 0.959 & 0.960 & 0.957 \\
\hline $\mathrm{f}^{\text {min }}$ & 0.246 & 0.254 & 0.926 & 0.923 & 0.937 \\
\hline
\end{tabular}

Table 4. Parameter distributions for the optimal calibrations for each of the different fitness functions used.

\begin{tabular}{|c|c|c|c|c|c|c|}
\hline & \multicolumn{2}{|c|}{ Max multiplier } & \multicolumn{2}{|c|}{ Max average } & \multicolumn{2}{c|}{ Max min } \\
\hline & Mean & stdev & mean & stdev & mean & Stdev \\
\hline$\alpha$ & 1.00 & 0.14 & 0.96 & 0.12 & 0.88 & 0.05 \\
\hline$\beta$ & 0.52 & 0.00 & 0.52 & 0.00 & 0.49 & 0.01 \\
\hline$\lambda$ & 3.10 & 0.27 & 3.50 & 0.38 & 1.50 & 0.01 \\
\hline$\eta$ & 0.37 & 0.00 & 0.52 & 0.07 & 0.33 & 0.01 \\
\hline$\tau_{1}$ & 0.51 & 0.00 & 0.56 & 0.00 & 0.82 & 0.04 \\
\hline$\tau_{2}$ & 0.71 & 0.07 & 0.57 & 0.00 & 0.38 & 0.05 \\
\hline
\end{tabular}

When we plot the data with the simulations results of the calibrated models, we see that the three different calibrations do not lead to main differences in the results (Figures 3-6). The patterns in the simulated data are smoother than the actual data. This is not strange since the simulated data is smoothed over 100 runs. We also see that the distribution of the collected tokens is more spread in the actual data than the simulated data (Figure 5).

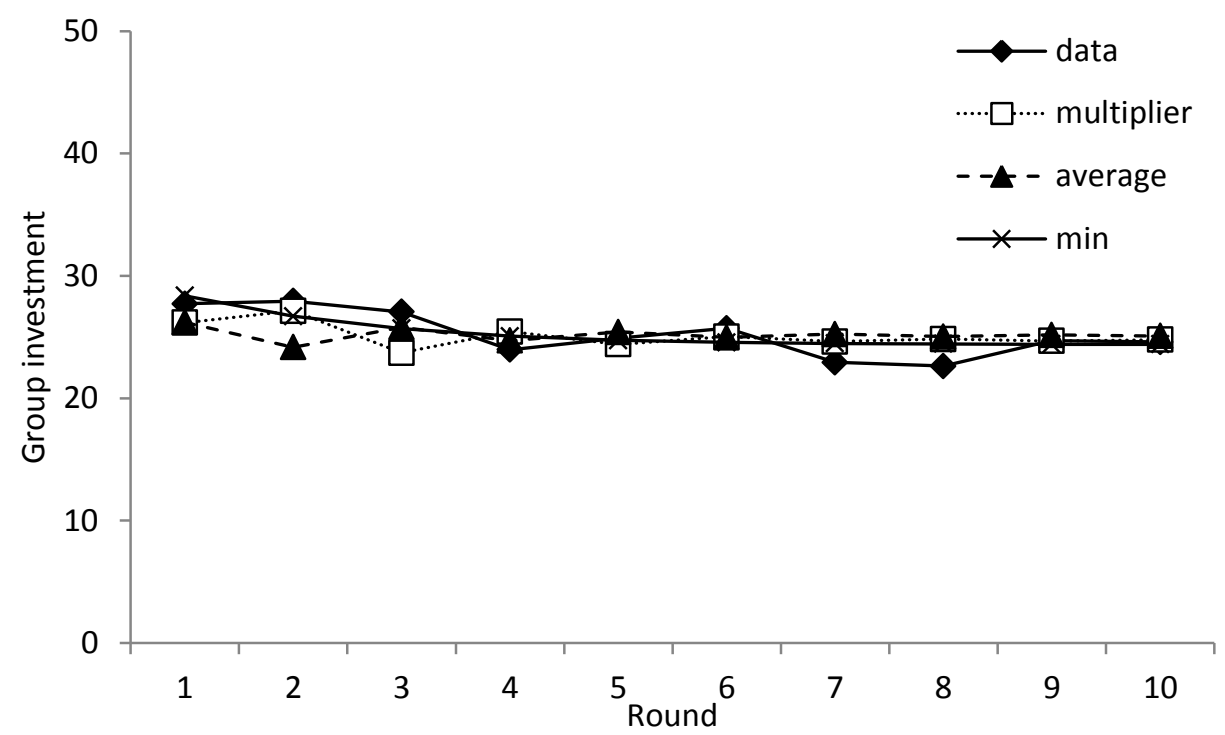

Fig 3. Average group investment levels per rounds for the data and the optimal calibrations using three different fitness score functions. 


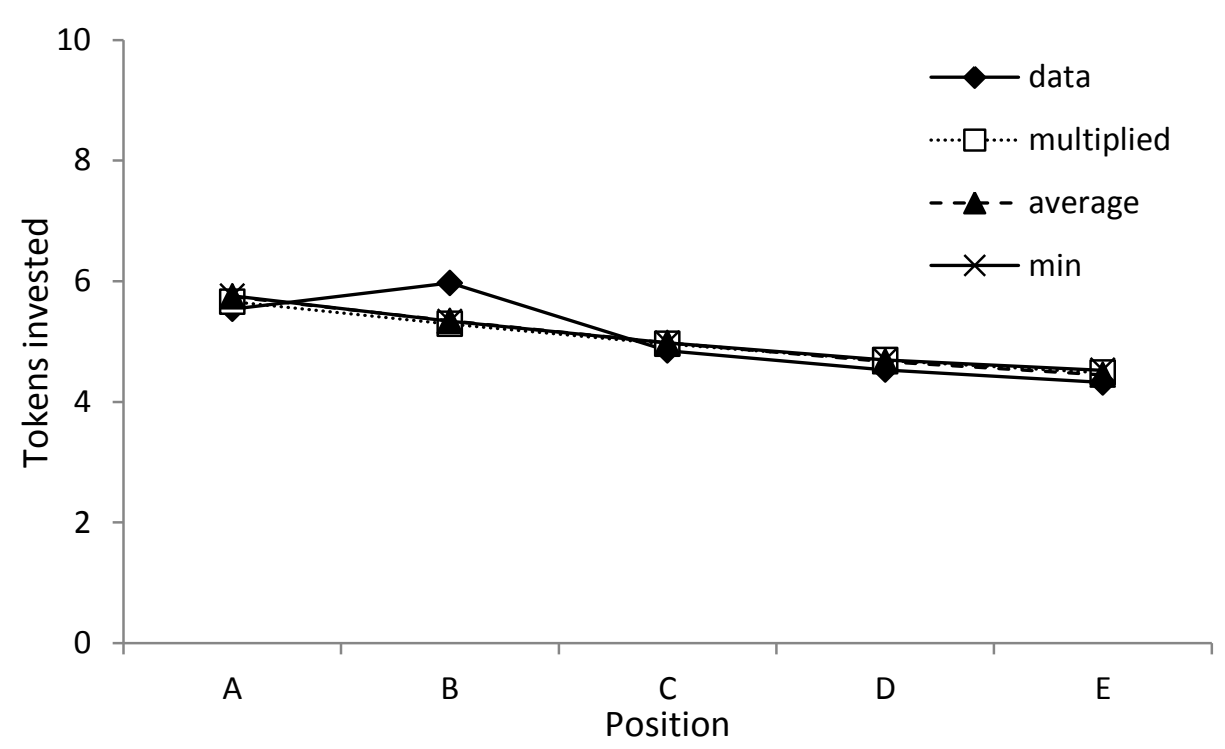

Fig.4. Average investment level per position for the data and the optimal calibrations using three different fitness score functions.

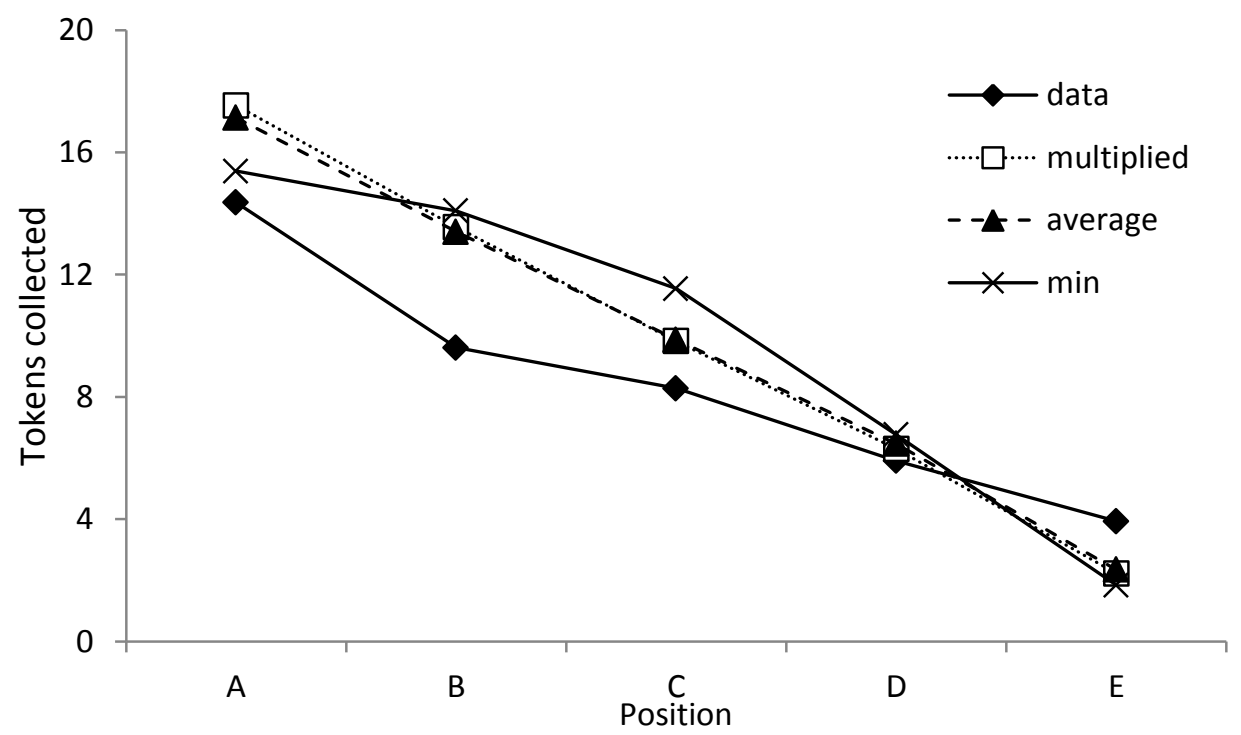

Fig 5. Average harvesting levels per position for the data and the optimal calibrations using three different fitness score functions. 


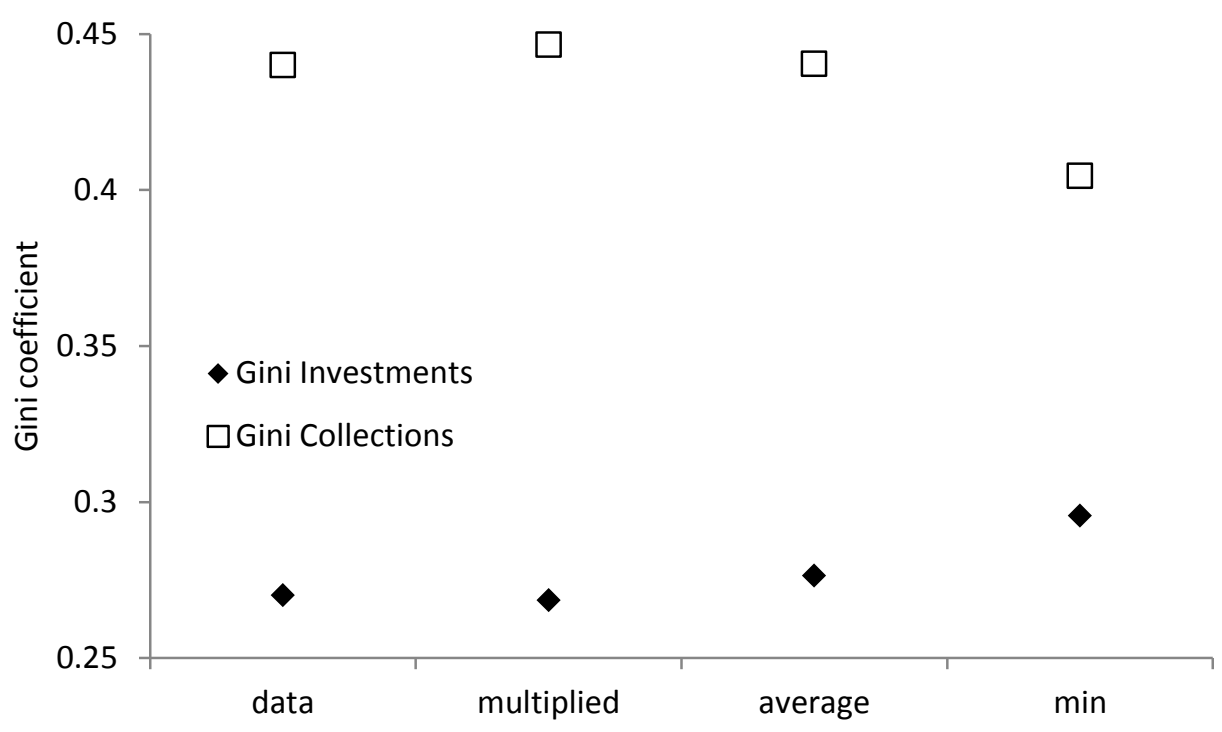

Fig 6. Average gini scores for investments and harvesting rate for the data and the optimal calibrations using three different fitness score functions.

Sensitivity analysis

A sensitivity analysis was performed to test the importance of particular assumptions of the model. For five cases we held parameters constant and analyzed the consequences for changes the mean of one of two parameters. For this analysis we uses only one fitness function, namely $\mathrm{f}^{\mathrm{mlt}}$, since the analysis above shows the robustness of the results for the different fitness functions.

The first case was the assumption of probabilistic choice (Figure 7). If agents always choose the option leading to the highest utility, the fitness score is dramatically reduced (from 0.8 to 0.2 ). With a probabilistic choice, we see that the model is not very sensitive to modest changes in the values of $\lambda$. If $\lambda$ is close to zero and agents make random decisions, we see that the fit is significantly reduced. Note that this means that a null model that makes random decisions from the options available (fitness $=0.66$ ) is doing a better job than the theoretical solution of the Nash equilibrium and the cooperative solution who have a fitness value of 0.008 .

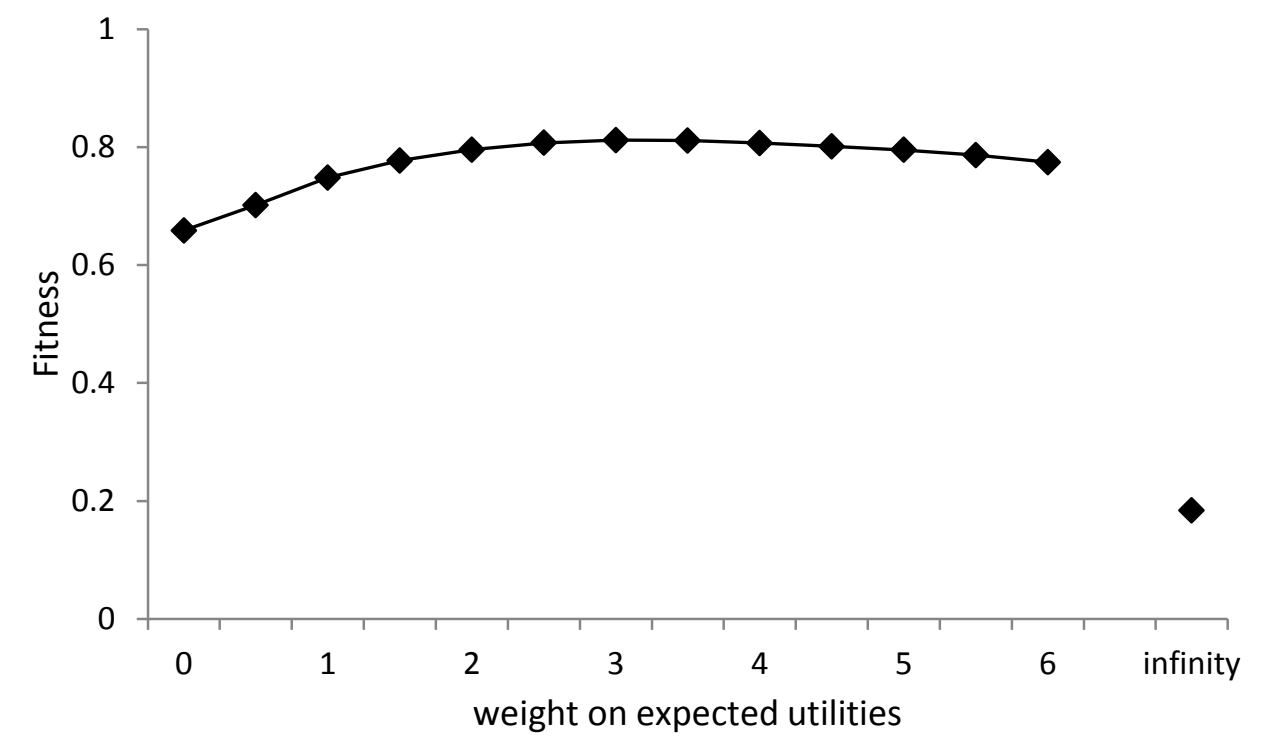

Fig 7. The effect of different values of $\lambda$ on the fitness of the model with the data.

If agents do not learn $\left(\tau_{1}=1\right)$, there is only a slight reduction of the performance. The performance of the model is insensitive to the learning rate of the share that upstream participants have extracted. The model is sensitive to changes in the learning rate of cooperation which affect the expectations of investments. If agents 
expect that observations in the current round will be the best estimate for the next round, the performance of the model drops. Hence agents will have a learning rate that takes into account observations of the last round and the long term trend. This avoid that agents will overreact to impulsive decisions made by others. In fact agents can forgive a selfish decision by not adjusting the cooperating expectations immediately in the next round.

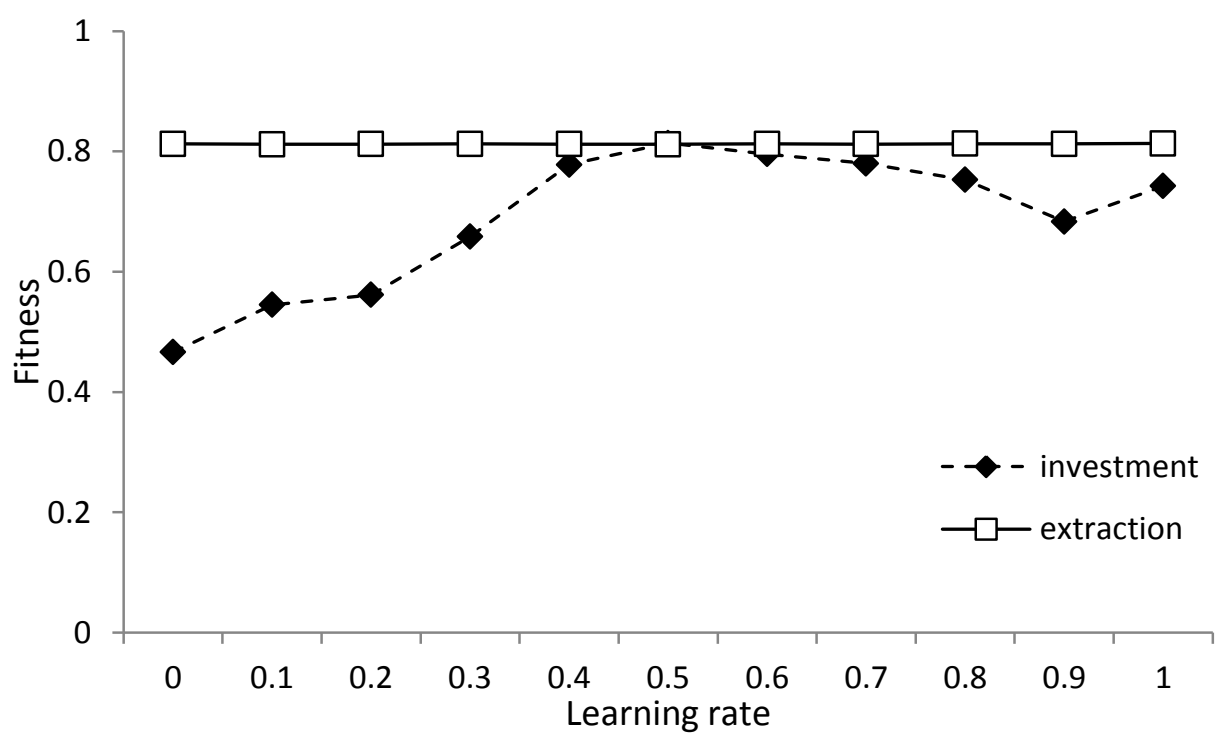

Fig 8. The effect of different learning rates $\tau_{1}$ and $\tau_{2}$.

When agents assume initially that other agents do not cooperate, the performance of the model drops to almost 0 . Agents will not cooperate and the investment levels will be close to 0 . The performance of the calibration is not much affected by the assumption of the initial expectation what other agents do. The best fit is when the expected level of cooperation is around $50 \%$.

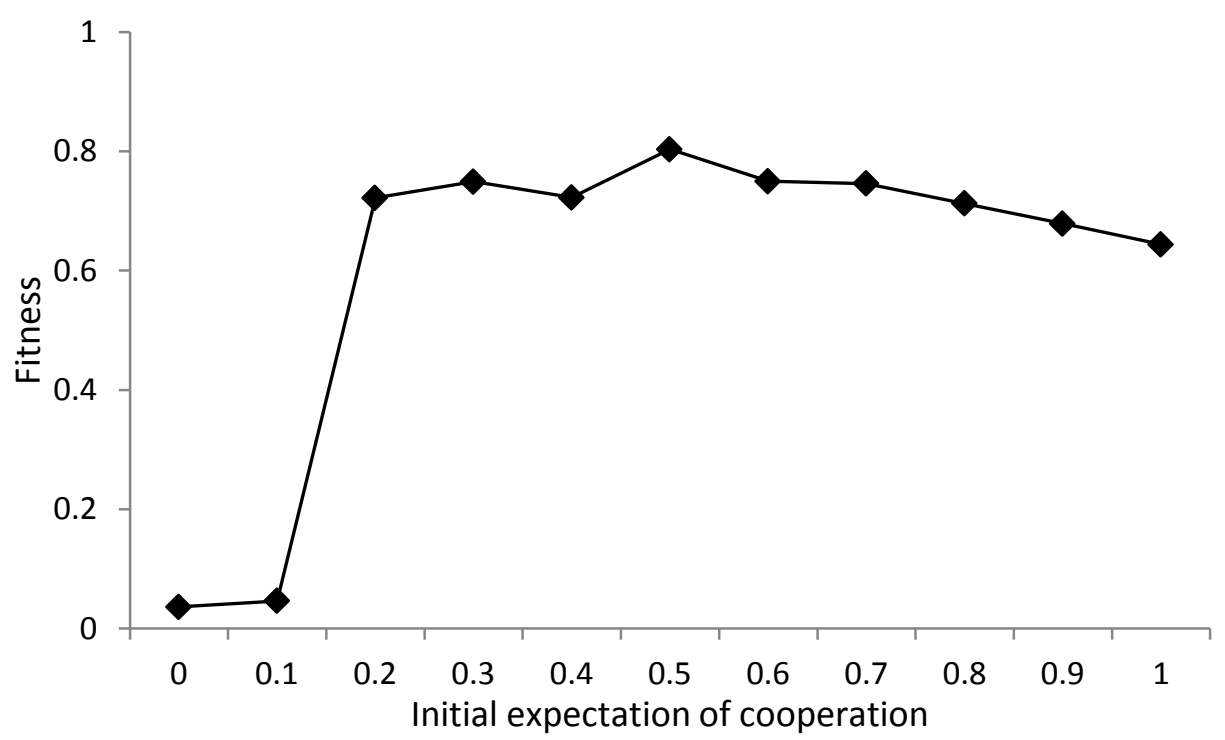

Fig. 10. The effect of different values of the expected level of cooperation by others.

Finally, if agents are assumed to be selfish and $\alpha$ and $\beta$ are equal to 0 the performance of the model is poor. The model performance starts to improve if $\beta$ is 0.5 or 0.6 and $\alpha$ is 0.5 or higher. The sensitivity analysis shows the importance of including other-regarding preferences within the model formulation. 


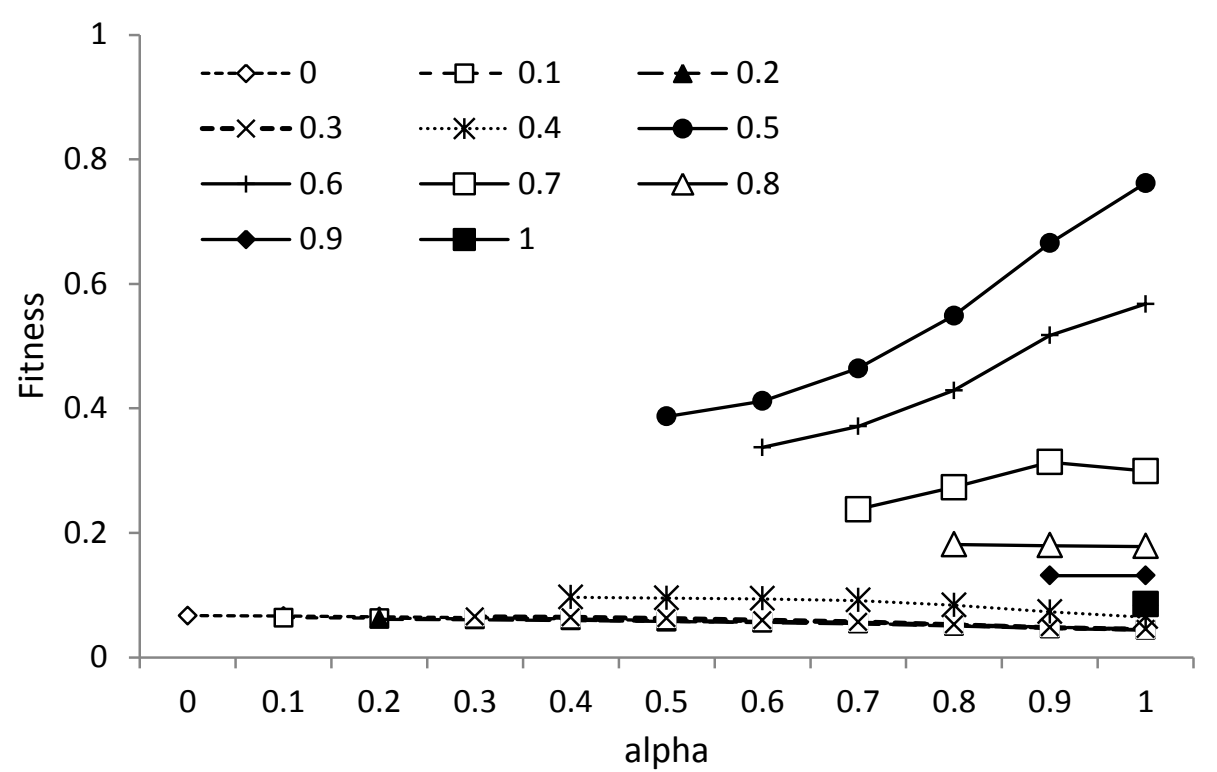

Fig 11. The effect of different values of $\alpha$ and $\beta$. The horizontal axis refers to different values of $\alpha$, while the different lines refer to different values of $\beta$.

\section{Simple model}

Based on the sensitivity analysis we define a simplified model. We assume $\tau_{1}$ is equal to 0.5 , $\tau_{2}$ is equal to 0 , and $\lambda$ equal to 3 , and $\eta$ is equal to 0.5 . We keep the other-regarding preferences as found in the calibration of the model. The fitness score is 0.801 and only slightly lower than the best score of 0.813 .

\section{Lessons Learned}

In this chapter a detailed calibration exercise of an agent-based model on field experimental data is discussed. The model was constructed to represent a general model of decision making in social dilemmas. It captures insights from behavioral games theory, namely assumptions on other-regarding preferences and learning. The model formulation was also affected by the insights from the statistical analysis of the particular field experiments.

The model did not aim to capture irrigation systems within a particular context, but decision making in a general context that resembles dilemmas irrigators have. With that in mind we use data from 6 villages and 2 student populations to create a data set of 32 groups. The aim of the model was to capture the general patterns coming out of these 32 groups.

The traditional model of rational selfish actors cannot explain the data, nor do the data support a cooperative solution. There is not a clear candidate model of the observations. Hence our model analysis contributes to the development of an alternative theory of collective action based on experimental data.

A challenge is to define the performance of a model. Different indicators provide information on the dynamics within the field experiments. These indicators capture distributions and averages at the group and individual level. We define five different patterns to capture the dynamics of the system.

Grimm et al. (2005) define pattern-oriented modeling to develop models that capture as many relevant patterns as possible. Typical pattern-oriented modeling exercises explore the parameter space to find parameter settings that capture the patterns within the uncertainty ranges. This has also been performed with experimental data (Janssen et al., 2009).

In this paper we decided to evaluate the model on the different patterns using optimization. We maximize the fit between data and simulations. Since there are different ways to aggregate information on performance on five different patterns, we tested the effects of these different fitness functions. The results are robust to the different fitness functions. 
The model was implemented in Netlogo and we could make use of the BehaviorSearch tool developed for Netlogo. This enables us to easily apply optimization to agent-based models. BehaviorSearch includes different optimization algorithms (random search, genetic algorithm, and hill climbing). We made use of genetic algorithms because of its proven performance on complex optimization problems. Since genetic algorithms do not always lead to the same results, we used 50 different runs for each optimization task.

In our sensitivity analysis we found particular assumptions of the model critical for the performance of the model. One critical assumption is the trembling hand, meaning that agents do not choose the option with highest utility. Another critical assumption is that agents are not selfish. Finally it is critical to assume that agents assume other agents have a modest level of cooperation. Based on our sensitivity analysis we could propose a simplified version of the model, which might be useful for other studies on irrigation systems and collective action.

Reflecting on the calibration analysis, we found a systematic sensitivity analysis to be helpful in understanding which aspects of the model are critical for the model performance. As such, we could propose a simplified model. The goal of this exercise is therefore not to find the specific parameter values, but to contribute to an alternative model of decision making in collective action situations. Our analysis confirms the critical aspects of other-regarding preferences and the trembling hand in decision making.

\section{References}

Camerer, C.F. 2003. Behavioral game theory: Experiments in strategic interaction. Princeton University Press, Princeton, NJ

Charness, G., and M. Rabin. 2002. Understanding social preferences with simple tests. Quarterly Journal of Economics 117: 817-869.

Fehr, E., and K.M. Schmidt. 1999. A theory of fairness, competition, and cooperation. The Quarterly Journal of Economics 114 (3): 817-868

Grimm, V.; E. Revilla, U. Berger, F. Jeltsch, W.M. Mooij, S.F. Railsback, H.H. Thulke, J. Weiner, T. Wiegand, and D.L. DeAngelis. 2005. Pattern-oriented modeling of agent-based complex systems: Lessons from ecology. Science 310: 987-991

Hunt R.C. 1988. Size and structure of authority in canal irrigation systems. Journal of Anthropological Research 44(4): 335-355.

Janssen, M.A., F. Bousquet, J.C. Cardenas, D. Castillo, K. Worrapimphong. 2012. Field experiments of irrigation dilemmas. Agricultural Systems 109: 65-75.

Janssen, M.A., N.P. Radtke, A. Lee. 2009. Pattern-oriented modeling of commons dilemma experiments, Adaptive Behaviour 17:508-523.

Lansing, J.S. 1991. Priests and programmers: Technologies of power in the engineered landscape of Bali. Princeton University Press, Princeton, NJ.

Ostrom, E. 1992. Crafting institutions for self-governing irrigation systems. ICS Press, San Francisco, CA.

Wittfogel, K.A. 1957. Oriental despotism: A comparative study of total power. Yale University Press, New Haven, CT.

1. 